\title{
Spiral galaxies with a central plateau in the gas velocity curve along the major axis ${ }^{\star, \star \star}$
}

\author{
L. Coccato, E. M. Corsini, A. Pizzella, and F. Bertola
}

\author{
Dipartimento di Astronomia, Università di Padova, vicolo dell'Osservatorio 2, 35122 Padova, Italy \\ e-mail: coccato@astro.rug.nl
}

Received 21 October 2004 / Accepted 17 May 2005

\begin{abstract}
We present the minor-axis kinematics of ionized gas and stars for a sample of 5 spiral galaxies, which are characterized by either a zero or a shallow gas velocity gradient along their major axis. The asymmetric velocity profiles observed along the minor axis of NGC 4064 and NGC 4189 can be explained as due to the presence of a bar. This is also the case of NGC 4178, where the innermost portion of the gaseous disk is nearly face on. In NGC 4424 and NGC 4941, we measured non-zero gas velocities only in the central regions along the minor axis, and gas velocities drop to zero at larger radii. This kinematic feature is suggestive of the presence of an orthogonally-rotating gaseous component, which is confined in the innermost regions (i.e. an inner polar disk) and needs to be confirmed with integral-field spectroscopy.
\end{abstract}

Key words. galaxies: kinematics and dynamics - galaxies: spirals - galaxies: structure

\section{Introduction}

In a series of papers (Bertola et al. 1999; Sarzi et al. 2000; Corsini et al. 2002, 2003; Coccato et al. 2004), we reported the case of spiral galaxies whose major-axis velocity curves of the ionized-gas and/or stellar component are characterized by a central plateau that is extended a few arcseconds. This uncommon feature has been also observed in two-dimensional velocity fields (e.g., Sil'chenko \& Afanaziev 2004). In some cases these galaxies exhibit a central velocity gradient along the minor axis with non-zero velocities confined in the central regions and dropping to zero at larger radii. In other cases the minoraxis velocity gradient extends all over the observed range or is missing (Coccato et al. 2004).

The presence of a major-axis velocity plateau together with a minor-axis central velocity gradient is the most interesting case, since it constitutes the kinematic signature of an inner polar disk (IPD hereafter). IPDs are small disks of gas and/or stars $(R \approx 300 \mathrm{pc})$, which are located in the center of lenticular and spiral galaxies and are rotating in a plane perpendicular to that of the main disk of their host. Most of these orthogonallydecoupled structures have been discovered in the last few years (see Corsini et al. 2003, and references therein) and only a number of IPDs have been studied in detail (Sil'chenko \& Afanaziev 2004).

\footnotetext{
* Based on observations carried out at the European Southern Observatory (ESO 67.A-0286 and 71.N-0229).

$\star \star$ Table 1 is only available in electronic form at the CDS via anonymous ftp to cdsarc.u-strasbg.fr (130.79.128.5) or via http://cdsweb.u-strasbg.fr/cgi-bin/qcat?J/A+A/440/107
}

Over the course of the past few years we have undertaken a program aimed at detecting IPDs using long-slit spectroscopic observations (Corsini et al. 2002, 2003; Coccato et al. 2004). In the present paper, which is an extension of our previous studies on IPDs, we present the analysis of a sample of 5 spiral galaxies, whose major-axis gas rotation curve shows either a remarkable zero-velocity plateau (NGC 4424 as measured by Kenney et al. 1996; and Rubin et al. 1999, NGC 4941 as discussed in this paper) or a shallow velocity gradient (NGC 4064, NGC 4178, NGC 4189 as found by Rubin et al. 1999). NGC 4064, NGC 4178, NGC 4189, and NGC 4424 belong to the sample of spiral galaxies measured by Rubin et al. (1999) to study the correlations between kinematic disturbances, location in the cluster, and tidal encounters of the galaxies in the Virgo cluster. NGC 4941 was found by us in a galaxy sample we studied for another purpose. These galaxies are promising candidates for our investigation because of their major-axis kinematic features. We therefore obtained spectra along their minor axis, in order to look for the possible presence of a velocity gradient. We present the ionized-gas and stellar kinematics of the sample galaxies in Sect. 2 and discuss our conclusions in Sect. 3.

\section{2. lonized-gas and stellar kinematics}

The long-slit spectroscopic observations of the sample galaxies were carried out with the New Technology Telescope (NTT) at the European Southern Observatory (ESO) in La Silla (Chile) on May 16, 2001 (run 1), April 25-30, 2003 (run 2), and April 30, 2003 (run 3). NTT mounted the ESO Multi-Mode 

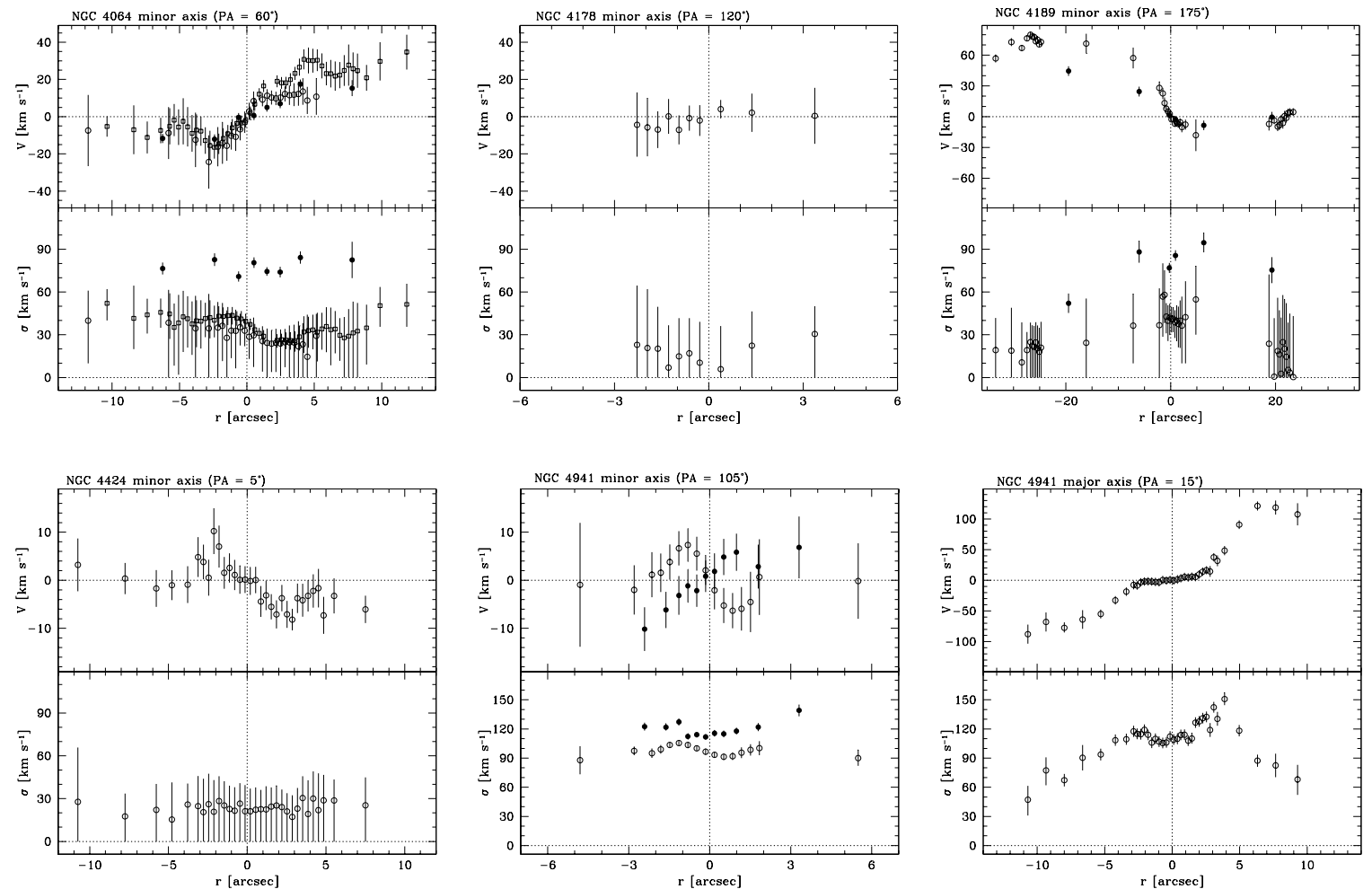

Fig. 1. Ionized-gas (open symbols) and stellar (filled circles) kinematics measured along the optical minor axis of NGC 4064, NGC 4178, NGC 4189, NGC 4424, and NGC 4941, and the optical major axis of NGC 4941. For NGC 4064, the open circles and open squares refer to gas kinematics obtained in runs 1 and 3, respectively.

Instrument in red medium-dispersion spectroscopic mode. The grating No. 6 with 1200 grooves $\mathrm{mm}^{-1}$ was mounted with the No. 36 Tektronix TK2048CCD in run 1 and with No. 62 MIT/LL 2048 CCD in run 2. The grating No. 7 with 600 grooves $\mathrm{mm}^{-1}$ was used in combination with the No. 62 MIT/LL $2048 \mathrm{CCD}$ in run 3 . In run 1 each $24 \times 24 \mu \mathrm{m}^{2}$ spectrum pixel corresponded to $0.32 \AA \times 0$.'270. In runs 2 and 3 a $2 \times 2$ binning was applied and each $30 \times 30 \mu \mathrm{m}^{2}$ spectrum pixel corresponded to $0.40 \AA \times 0.332$ and $0.82 \AA \times 0 . ' 332$, respectively. The slit was 1.'0 wide and 5.5 long and gave an instrumental $F W H M$ of $1.1 \AA$ in run $1(6400-7020 \AA)$ and 2 (4830-5470 $\AA$ ), and $2.2 \AA$ in run 3 (5850-7150 A). Typical seeing $F W H M$ was $1^{\prime \prime} .8$ in run 1 and 0.9 in runs 2 and 3 as measured by the ESO Differential Image Motion Monitor.

For each sample galaxy, we took a spectrum with the slit along the minor axis (NGC $4064 \mathrm{PA}=60^{\circ}, \mathrm{NGC} 4178$ $\mathrm{PA}=120^{\circ}, \mathrm{NGC} 4189 \mathrm{PA}=175^{\circ}, \mathrm{NGC} 4424 \mathrm{PA}=5^{\circ}$, and NGC $4941 \mathrm{PA}=105^{\circ}$ ). For NGC 4941 a major-axis spectrum $\left(\mathrm{PA}=15^{\circ}\right)$ was obtained, too. The position angles of the major and minor axis were chosen according to RC3 and therefore relate to the orientation of the outermost isophotes. Integration time of the galaxy spectra was typically $5400 \mathrm{~s}$ and split into different exposures to deal with cosmic rays. In run 2 we obtained spectra of some giant stars with spectral type ranging from late- $\mathrm{G}$ to early- $\mathrm{K}$ to be used as templates in measuring the minor-axis stellar kinematics of NGC 4064, NGC 4189, and NGC 4941. Arc lamp spectra were taken before and/or after every object exposure to allow an accurate wavelength calibration.
The basic data reduction and the measurement of the ionized-gas and stellar kinematics was performed as in Corsini et al. (2003). The line-of-sight velocity and velocity dispersion profiles we measured for the gaseous and stellar component of the sample galaxies are presented in Fig. 1 and values are reported in Table 1. The line-of-sight velocities are the observed ones after subtracting the systemic velocities derived as center of symmetry of the gas velocity profile and without applying any correction for galaxy inclination. We measured $c z=968 \pm 5 \mathrm{~km} \mathrm{~s}^{-1}$ for NGC 4064, $c z=390 \pm 5 \mathrm{~km} \mathrm{~s}^{-1}$ for NGC 4178, $c z=2086 \pm 5 \mathrm{~km} \mathrm{~s}^{-1}$ for NGC 4189, $c z=$ $454 \pm 5 \mathrm{~km} \mathrm{~s}^{-1}$ for NGC 4424, $c z=1120 \pm 5 \mathrm{~km} \mathrm{~s}^{-1}$ for NGC 4941. The line-of-sight velocity dispersions are corrected for instrumental velocity dispersion.

The following kinematic features are noteworthy in the galaxies of our sample:

1. The major-axis velocity gradient of the ionized gas is zero in the central regions of NGC 4189 (Rubin et al. 1999), NGC 4424 (Kenney et al. 1996; Rubin et al. 1999), and NGC 4941 (Fig. 1). It is shallower than that measured at larger radii in NGC 4064 and NGC 4178 (Rubin et al. 1999). Further out, the gas velocity remains low ( $\$ 20 \mathrm{~km} \mathrm{~s}^{-1}$, NGC 4064) or it increases to the last observed radius (NGC 4178, NGC 4189, NGC 4424, and NGC 4941).

2. Non-zero gas velocities are measured along the minor axis of all the galaxies except for NGC 4178 (Fig. 1), in spite of what would be expected if the gas traced the circular 
velocity in the disk plane. The ionized-gas velocity profile measured along the optical minor axis of NGC 4064 and NGC 4189 is strongly asymmetric. The gas velocity rises to a maximum and remains almost constant out to the last observed point on the receding side. On the contrary, it drops to zero at large radii on the approaching side. The non-zero gas velocities are confined in the central regions along the minor axis of NGC 4424 and NGC 4941, where the gas velocity curve shows a steep gradient. The gas velocity increases to a maximum of $\approx 10 \mathrm{~km} \mathrm{~s}^{-1}$ in the inner few arcsec, while further out it decreases to zero. Our measurements along the minor axis of NGC 4424 are in agreement, within errors, with those by Kenney et al. (1996), which are characterized by a lower spatial sampling. A zero velocity gradient is observed for the ionized-gas component along the minor axis of NGC 4178. In all the galaxies the gas velocity dispersion remains low $\left(\$ 50 \mathrm{~km} \mathrm{~s}^{-1}\right)$, except for NGC 4941 where it reaches $\approx 100 \mathrm{~km} \mathrm{~s}^{-1}$ in the inner $\sim 5^{\prime \prime}$.

3. Non-zero velocities are measured along the minor axis of NGC 4064, NGC 4189, and NGC 4941 for the stellar component, too. The stars rotate slower than gas and have a larger velocity dispersion $\left(\approx 80 \mathrm{~km} \mathrm{~s}^{-1}\right)$ in NGC 4064 and NGC 4189. Finally, in NGC 4941 stars are counterrotating with respect to gas. They show a maximum observed velocity of $\approx 10 \mathrm{~km} \mathrm{~s}^{-1}$ and a constant velocity dispersion of $\approx 120 \mathrm{~km} \mathrm{~s}^{-1}$.

\section{Discussion and conclusions}

We have reported the ionized-gas and stellar kinematics along the disk minor axis of 5 spiral galaxies, which are known to have either a zero or a shallow gas velocity gradient along the disk major axis.

NGC 4064, NGC 4178, and NGC 4189 host a large-scale bar (RC3, but see also CAG). Qualitatively, the minor-axis gas kinematics can be explained as due to non-circular (e.g., Athanassoula 1992) or off-plane (e.g., Friedli \& Benz 1993) motions induced on the gaseous component by the tumbling triaxial potential of the bar. The innermost portion of the gaseous disk of NGC 4178 is nearly face-on since a zero velocity gradient is observed along both major and minor axes. Bar can account also for the stellar velocity gradient observed along the minor axis of NGC 4064 and NGC 4189.

In spite of the morphological classification in RC3, NGC 4424 is an unbarred galaxy. Kenney et al. (1996) first pointed out the presence of strong non-circular gas motions and/or rotation in a plane different from that of the outer stellar disk and attributed it to a recent merger. We suggest that the ionized-gas kinematics measured for $|r| \lesssim 5^{\prime \prime}$ is consistent with the presence of an IPD.

No large-scale bar has been observed in the early-type spiral NGC 4941 (Greusard et al. 2000), which is a Seyfert 2 galaxy (Stauffer 1982). The peculiar gas kinematics can be associated to the extended emission-line nuclear structure found by Martini et al. (2003), which is roughly aligned with the galaxy minor axis and can be explained as due to outflowing photoionized gas. However, this does not preclude that such a gaseous component is an IPD. It is worth noting that an IPD of gas and stars has been observed in the Seyfert 2 galaxy NGC 4698 (Bertola et al. 1999; Bertola \& Corsini 2000; Pizzella et al. 2002). Moreover NGC 4941 hosts a nuclear bar, which is almost aligned with the galaxy major axis $(\triangle \mathrm{PA} \approx$ $\left.15^{\circ}\right)$ and confined to the region where the kinematic peculiarities are measured (Greusard et al. 2000). Therefore the observed kinematics is consistent with the presence of gas moving onto anomalous orbits in the tumbling potential of the nuclear bar. This is also supported by the presence of gas in retrograde motion relative to the stars along the disk minor axis (i.e., in a direction close to the bar minor axis), where the anomalous orbits are expected to be highly inclined with respect to the rotation axis of the bar (see Friedli \& Benz 1993, for details). In this scenario the gas is settled into a stable configuration forming a strongly warped disk, whose innermost portion corresponds to an IPD. This is the case of NGC 2217 (Bettoni et al. 1990), which is characterized by the same gaseous and stellar kinematics as NGC 4941.

We conclude that NGC 4424 and NGC 4941 are good candidates for following up with integral-field spectroscopy in order to address the presence of an IPD. These nuclear structures are remarkable enough to require remarkable evidence, and only the measurement of the two-dimensional velocity field at high spatial resolution can fully constrain the size and orientation of IPDs and will allow us to get clues about how they have been formed.

\section{References}

Athanassoula, E. 1992, MNRAS, 259, 345

Bettoni, D., Fasano, G., \& Galletta, G. 1990, AJ, 99, 1789

Bertola, F., \& Corsini, E. M. 2000, In Galaxy Dynamics: from the Early Universe to the Present, ed. F. Combes, G. A. Mamon, \& V. Charmandaris (San Francisco: ASP), ASP Conf. Ser., 197, 115

Bertola, F., Corsini, E. M., Vega Beltrán, J. C., et al. 1999, ApJ, 519, L127

Coccato, L., Corsini, E. M., Pizzella, A., et al. 2004, A\&A, 416, 507

Corsini, E. M., Pizzella, A., \& Bertola, F. 2002, A\&A, 283, 488

Corsini, E. M., Pizzella, A., Coccato, L., \& Bertola, F. 2003, A\&A, 408,873

de Vaucouleurs, D., de Vaucouleurs, A., Corwin, H. G., Jr., et al. 1991, Third Reference Catalog of Bright Galaxies (New York: SpringerVerlag), RC3

Friedli, D., \& Benz, W. 1993, A\&A, 268, 65

Greusard, D., Friedli, D., Wozniak, H., Martinet, L., \& Martin, P. 2000, A\&AS, 145, 425

Kenney, J. D. P., Koopmann, R. A., Rubin, V. C., \& Young, J. S. 1996, AJ, 111, 152

Martini, P., Regan, M. W., Mulchaey, J. S., \& Pogge, R. W. 2003, ApJS, 146, 353

Pizzella, A., Corsini, E. M., Morelli, L., et al. 2002, ApJ, 573, 131

Rubin, V. C., Waterman, A. H., \& Kenney, J. D. P. 1999, AJ, 118, 263

Sandage, A., \& Bedke, J. 1994, The Carnegie Atlas of Galaxies (Washington: Carnegie Institution), CAG

Sarzi, M., Corsini, E. M., Pizzella, A., et al. 2000, A\&A, 360, 439

Sil'chenko, O. K., \& Afanasiev, V. L. 2004, AJ, 127, 2641

Stauffer, J. R. 1982, ApJ, 262, 66 